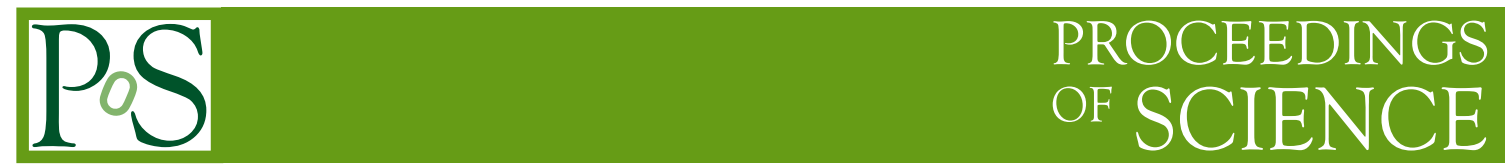

\title{
Neutrinos, Flavour and CP Violation
}

\section{Steve F. King*}

Physics and Astronomy, University of Southampton, Southampton, SO17 1BJ, U.K.

E-mail: kingesoton.ac.uk

In this talk we shall discuss models of neutrino masses, mixing and CP violation based on discrete family symmetry, following the measurement of the reactor angle in 2012. Various different approaches to model building are described, denoted as direct, semi-direct and indirect, and the pros and cons of each approach discussed. Particular examples of direct models based on big groups such as $\Delta\left(6 n^{2}\right)$ will be discussed, as well as indirect models based on small groups such as $A_{4}$ involving sequential dominance, exemplified by the $\mathrm{A}$ to $\mathrm{Z}$ of Flavour with Pati-Salam. Discrete family symmetries could originate from F-theory.

18th International Conference From the Planck Scale to the Electroweak Scale 25-29 May 2015

Ioannina, Greece

*Plenary talk at Planck 2015, Ionaninna, Greece. 


\section{Introduction}

We begin with a brief reminder about neutrino mass and mixing. The reactor angle was unmeasured before 2012 but is now measured to incredible accuracy: $\theta_{13} \approx 8.4^{\circ} \pm 0.2^{\circ}$ (see [1] and references therein). The other angles are determined from global fits [2, 3, 4] to be: $\theta_{12} \approx 34^{\circ} \pm 1^{\circ}$ and $\theta_{23} \approx 45^{\circ} \pm 3^{\circ}$, and first hints of the CP-violating (CPV) phase $\delta \sim-\pi / 2$ have been reported, however with a large error $\pm \pi / 3$. The meaning of the angles is given in Fig.1. Two possible mass squared orderings are possible as explained in Fig.2. The above quoted angles are extracted from the global fits which are displayed in Fig. 3 for the normal ordering case.

It is interesting to consider the history of model building in the light of these experimental developments. Following the confirmation of large atmospheric and solar mixing angles in 2002, the state of model building was summarised in the review [6]. The models discussed there involved sequential dominance (SD) which generally predicted a normal neutrino mass hierarchy with a reactor angle $\theta_{13} \lesssim m_{2} / m_{3}$, close to the existing limits [6]. Shortly afterwards it became very popular to consider models based on tri-bimaximal lepton mixing involving a zero reactor angle, enforced by discrete family symmetry as summarised in the reviews [7, 8]. Following the measurement of the reactor angle in 2012, it was shown how such discrete family symmetry models could be modified to account for the observed reactor angle as discussed in the reviews $[9,10]$. This talk summarises the developments discussed in those reviews and subsequently.

\section{The Standard Model Puzzles}

Even though the Standard Model (SM) is essentially complete, following the Higgs boson discovery, we are far from satisfied since it offers no solutions to the cosmological puzzles of matter-antimatter asymetry, dark matter and dark energy. It therefore cannot be the final answer. In addition it leaves three unresolved puzzles in its wake:

The origin of mass - the origin of the Higgs vacuum expectation value, its stability under radiative corrections, and the solution to the hierarchy problem (most urgent problem of LHC).

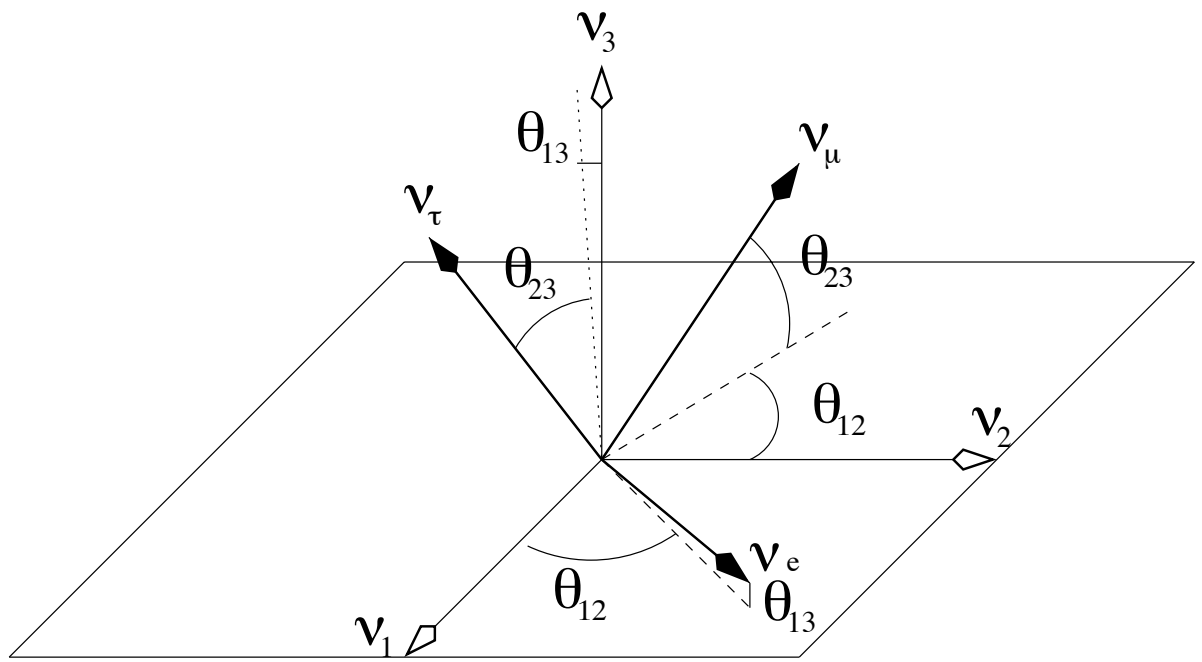

Figure 1: Neutrino mixing angles (assuming zero CP violation) may be represented as Euler angles relating the charged lepton mass basis states $\left(v_{e}, v_{\mu}, v_{\tau}\right)$ to the mass eigenstate basis states $\left(v_{1}, v_{2}, v_{3}\right)$. 


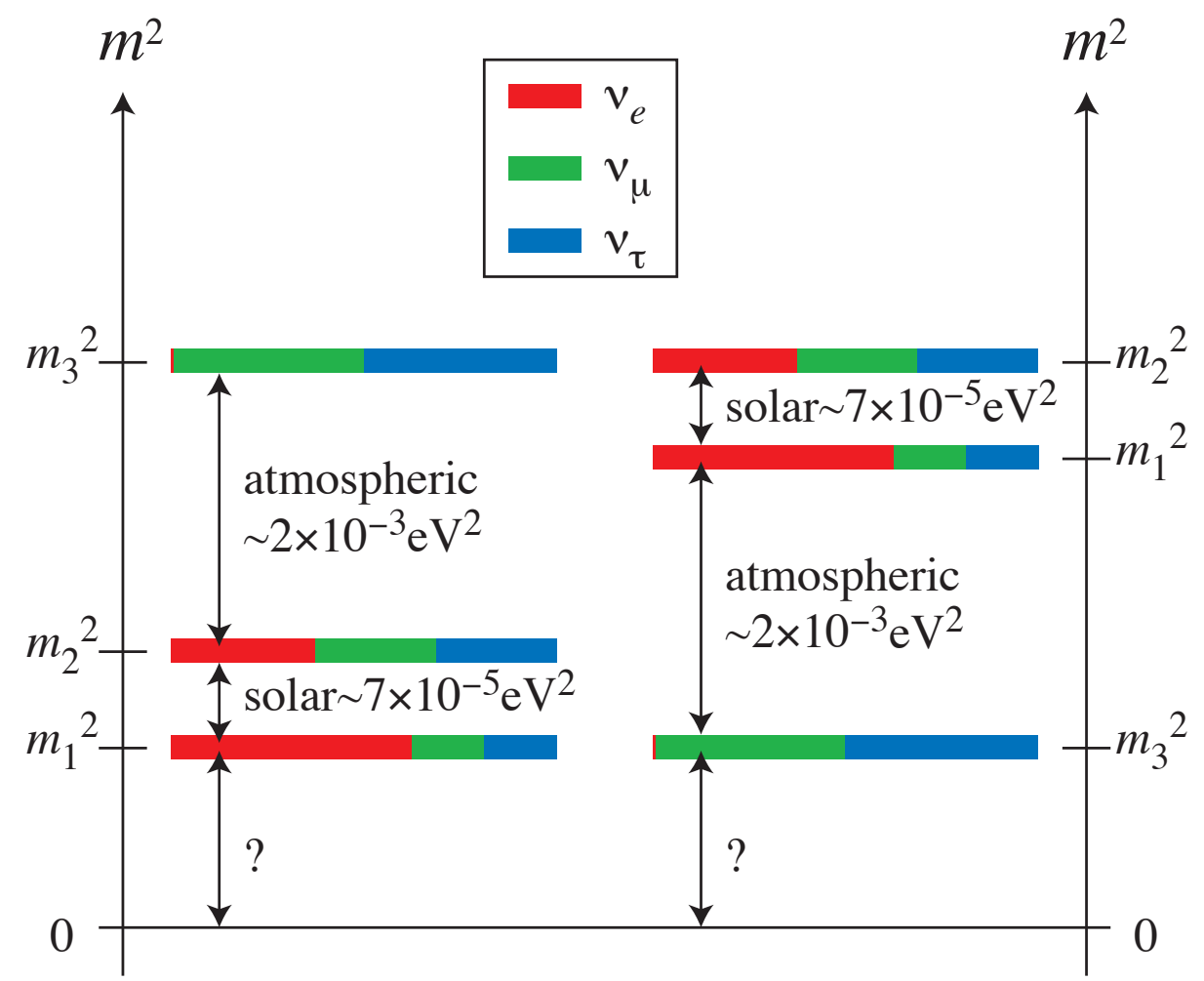

Figure 2: The probability that a particular neutrino mass state $v_{i}$ with mass $m_{i}$ contains a particular charged lepton mass basis state $\left(v_{e}, v_{\mu}, v_{\tau}\right)$ is represented by colours. The left and right panels of the figure are referred to as normal or inverted mass squared ordering, respectively. The value of the lightest neutrino mass is presently unknown.

The quest for unification - the question of whether the three known forces of the standard model may be related into a grand unified theory, and whether such a theory could also include a unification with gravity.

The problem of flavour - the problem of the undetermined fermion masses and mixing angles (including neutrino masses and lepton mixing angles) together with the $\mathrm{CP}$ violating phases, in conjunction with the observed smallness of flavour changing neutral currents and very small strong $\mathrm{CP}$ violation. In particular the unknown origin of the extremely small neutrino masses for all three families may offer a clue as to what lies beyond the SM.

The differences between quark and lepton mixing may also offer clues concerning the flavour problem. Certainly the flavour problem has now become much richer, following the discovery of neutrino mass and mixing, so we shall discuss more about this.

Before proceeding to discuss the flavour problem in more detail, we disgress slightly to discuss an alternative point of view that frequently is voiced and that came up again at this Workshop. Namely, all that is required for neutrino masses is to add two or three right-handed neutrinos with zero Majorana mass due to a conserved $B-L$, and Yukawa couplings for all neutrino families of about $10^{-11}$ and that no new physics beyond this is required. However this conservative point of view involves a new mystery, namely why the third family Yukawa couplings are of order unity for the top quark, and not very small for the bottom quark and $\tau$ lepton, but are of order $10^{-11}$ for the 

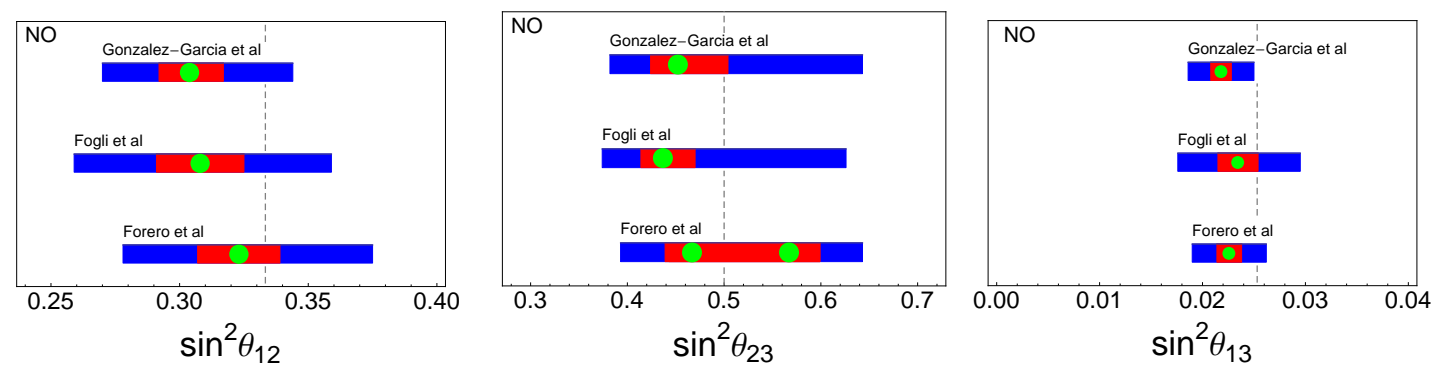

Figure 3: Global fits $[2,3,4]$ to the lepton mixing angles for the case of normal neutrino mass squared ordering. The green dots are the best fit points, the red (blue) areas indicate the one (three) sigma ranges. The dashed lines indicate tri-bimaximal-Cabibbo (TBC) mixing [5], namely the values: $s_{12}^{2}=1 / 3, s_{23}^{2}=1 / 2$ $s_{13}^{2}=\theta_{C}^{2} / 2$. The Fogli et al fits do not include the latest Daya Bay results, whereas the other two do. This is a modified version of a figure provided by Stefano Morisi.

third family of neutrinos. The see-saw mechanism [11], i.e. large third family neutrino Yukawa couplings, with physical neutrino masses suppressed by heavy right-handed Majorana masses with $B-L$ broken at a high scale, provides an elegant solution to this mystery, and opens the door to leptogenesis. However, the see-saw mechanism by itself does not account for the observed large lepton mixing, so we need to go further.

It has been one of the long standing goals of theories of particle physics beyond the Standard Model to predict quark and lepton masses and mixings. With the discovery of neutrino mass and mixing, this quest has received a massive impetus. Indeed, perhaps the greatest advance in particle physics over the past decade has been the discovery of neutrino mass and mixing involving large mixing. The largeness of the lepton mixing angles contrasts with the smallness of the quark mixing angles, and this observation, together with the smallness of neutrino masses, provides new and tantalising clues in the search for the origin of quark and lepton flavour. For example, it is intruiging that the smallest lepton mixing may be related to the largest quark mixing, $\left|U_{e 3}\right| \approx \theta_{C} / \sqrt{2}$ where $\theta_{C}$ is the Cabibbo angle, although this relation is in tension with the latest Daya Bay results. The quest to understand the origin of the three families of quarks and leptons and their pattern of masses and mixing parameters is called the flavour puzzle, and motivates the introduction of family symmetry. In particular, as we shall see, lepton mixing provides a motivation for discrete family symmetry, which will form the central part of this review. As we shall also see, such theories demand a high precision knowledge of the lepton mixing angles, beyond that currently achieved.

The SM does not address the following questions which loosely define the flavour problem:

- Why are there three families of quarks and leptons?

- Why are all charged fermion masses so hierarchical with down-type quark masses being of the same order as charged lepton masses, and up-type quark masses are much more hierarchical?

- Why are at least two neutrino masses not very hierarchical?

- What is the origin of the neutrino mass?

- Why are neutrino masses so tiny compared to charged fermion masses? 
- What is the origin of fermion mixing (both CKM and PMNS matrices)?

- Why are CKM mixing angles smaller than PMNS mixing angles apart from the Cabibbo angle which is of the same order as the reactor angle?

- What is the origin of $\mathrm{CP}$ violation in the quark (and lepton) sectors?

Both the CKM and the PMNS mixing matrices are parameterised by:

$$
\left(\begin{array}{ccc}
c_{12} c_{13} & s_{12} c_{13} & s_{13} e^{-i \delta} \\
-s_{12} c_{23}-c_{12} s_{13} s_{23} e^{i \delta} & c_{12} c_{23}-s_{12} s_{13} s_{23} e^{i \delta} & c_{13} s_{23} \\
s_{12} s_{23}-c_{12} s_{13} c_{23} e^{i \delta} & -c_{12} s_{23}-s_{12} s_{13} c_{23} e^{i \delta} & c_{13} c_{23}
\end{array}\right)
$$

where $\delta$ is the CP violating phase in each sector (quark and lepton) and $s_{13}=\sin \theta_{13}$, etc. with (very) different angles for quarks and leptons. In the quark sector the mixing angles are all small, with

$$
s_{12}=\lambda, s_{23} \sim \lambda^{2}, s_{12} \sim \lambda^{3}
$$

where $\lambda=0.226 \pm 0.001$ is the Wolfenstein parameter. The CP violating phase in the quark sector is roughly $\delta \sim(\pi / 2) / \sqrt{2} .{ }^{1}$ The lepton mixing angles, given in Fig. 3 and discussed in further in the next section, must arise in conjunction with the mechanism responsible for the smallness of neutrino mass, which however is unknown. In the case of Majorana neutrinos, the PMNS matrix also involves the phase matrix: $\operatorname{diag}\left(1, e^{i \frac{\alpha_{21}}{2}}, e^{i \frac{\alpha_{31}}{2}}\right)$ which post-multiplies the above matrix.

\section{Neutrino Mass Models}

\subsection{The open questions from neutrino physics}

Despite the great progress coming from neutrino oscillation experiments there are still some outstanding questions. Are the lepton mixing angles consistent with TBC mixing? If not then is the atmospheric angle in the first or second octant? What is the leptonic CP violating phase $\delta$ ? Is the current hint $\delta \sim-\pi / 2$ going to hold up? Maybe there is no CP violation in the lepton sector? Are neutrino mass squared ordered normally or inverted? ${ }^{2}$ What is the lightest neutrino mass? Are neutrino masses Majorana or Dirac in nature? Many neutrino experiments are underway or in the planning stages to address these questions such as T2K, NOvA, Daya Bay, JUNO, RENO, KATRIN, DUNE and many neutrinoless double beta decay experiments running and planned.

\subsection{Road Map of Neutrino Mass Models}

Everyone can invent her or his personal roadmap of neutrino mass models, one example being that shown in Fig.4. The blue boxes contain experimental questions and the red boxes possible theoretical consequences. Since no new physics on the right-hand side has yet emerged from the LHC, in this talk we shall focus on the see-saw mechanism [11] with right-handed neutrinos.

\footnotetext{
${ }^{1}$ Interestingly, in the original KM parametrisation, the quark CP violating phase is $\delta \sim \pi / 2$. Similarly, $\alpha \sim \pi / 2$ in the standard unitarity triangle representing the orthogonality of the 1st and 3rd columns of the CKM matrix.

${ }^{2}$ It is common but incorrect to refer to this question as the "neutrino mass hierarchy" since the "ordering" question is separate from whether neutrinos are hierarchical in nature or approximately degenerate, which is to do with the lightest neutrino mass.
} 


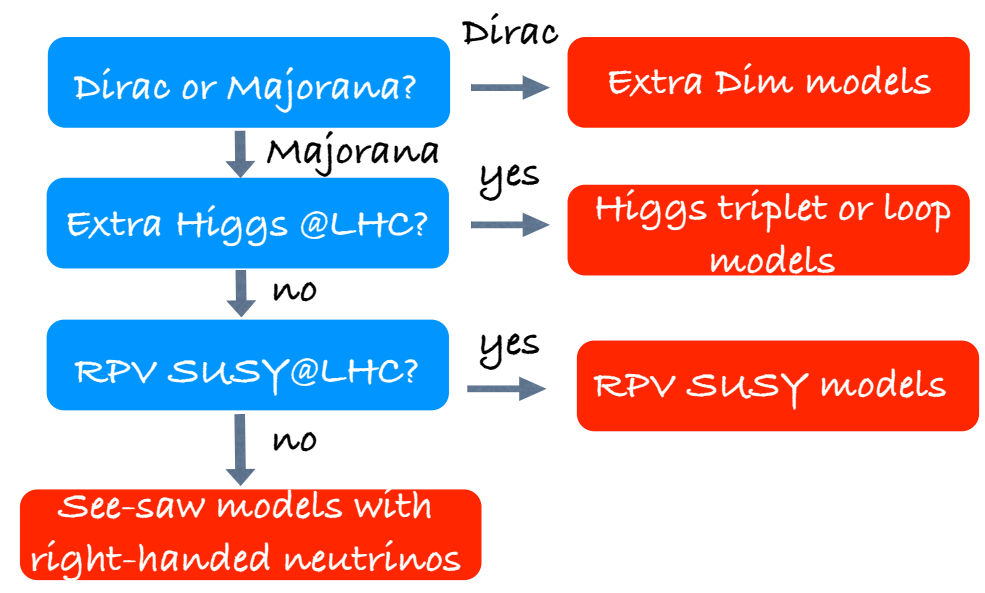

Figure 4: Roadmap of neutrino mass models.

\subsection{See-saw mechanism with right-handed neutrinos}

Both the number of species and the mass spectrum of right-handed (or sterile) neutrinos is completely unknown [12]. As shown in Fig.5 the mass spectrum can cover the whole range with different physical consequences as indicated. It is one of the goals of neutrino physics to determine this spectrum.

\subsection{See-saw mechanism and sequential dominance}

Suppose that the Standard Model is supplemented by just three right-handed neutrinos with masses in the classic see-saw range $\mathrm{TeV}$ to $\mathrm{M}_{\mathrm{GUT}}$ in Fig.5. Then the light neutrino mass matrix emerges from the see-saw formula [11],

$$
m^{v}=-m^{D} M_{R}^{-1}\left(m^{D}\right)^{T},
$$

where $m^{v}$ is the the light effective left-handed Majorana neutrino mass matrix (i.e. the physical neutrino mass matrix), $m^{D}$ is the Dirac mass matrix in LR convention and $M_{R}$ is the (heavy) Majorana mass matrix.

In this case it is possible to implement the see-saw mechanism using sequential dominance (SD) mechanism [13], in the basis, $M_{R}=\operatorname{diag}\left(M_{\mathrm{atm}}, M_{\mathrm{sol}}, M_{\mathrm{dec}}\right)$ where the Dirac mass matrix is constructed from three columns $m^{D}=\left(m_{\mathrm{atm}}^{D}, m_{\mathrm{sol}}^{D}, m_{\mathrm{dec}}^{D}\right)$,

$$
\frac{m_{\mathrm{atm}}^{D}\left(m_{\mathrm{atm}}^{D}\right)^{T}}{M_{\mathrm{atm}}}>\frac{m_{\mathrm{sol}}^{D}\left(m_{\mathrm{sol}}^{D}\right)^{T}}{M_{\mathrm{sol}}} \gg \frac{m_{\mathrm{dec}}^{D}\left(m_{\mathrm{dec}}^{D}\right)^{T}}{M_{\mathrm{dec}}} .
$$

SD immediately predicts a normal neutrino mass hierarchy $m_{3}>m_{2} \gg m_{1}$ since the lightest physical neutrino mass $m_{1}$ is much smaller than the others since the corresponding right-handed neu- 


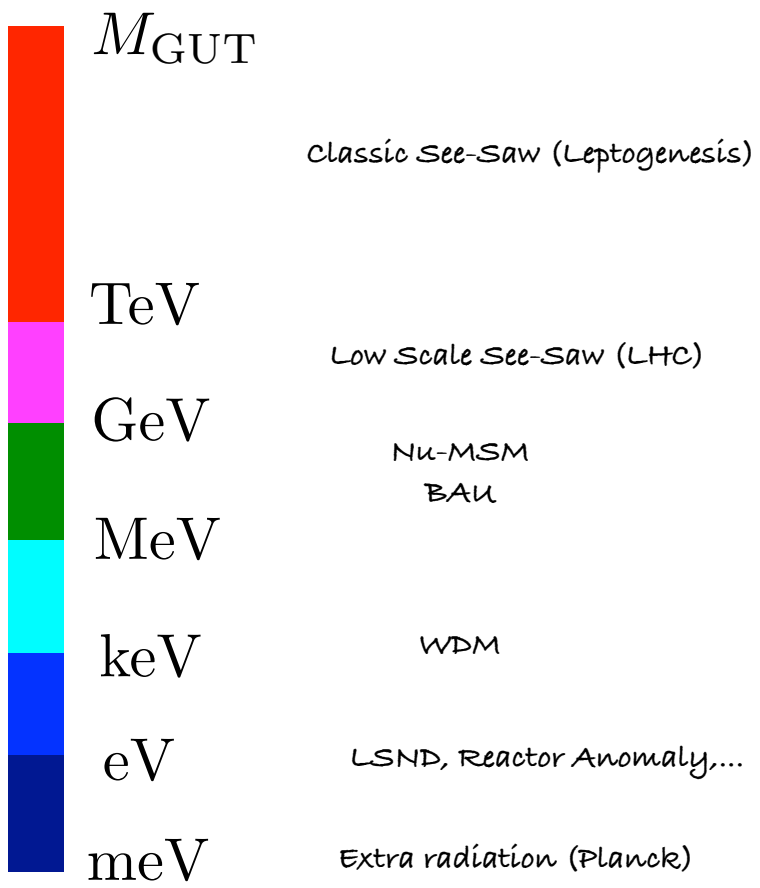

Figure 5: Possible mass spectrum of right-handed (or sterile) neutrinos corresponding to the physical implications as shown.
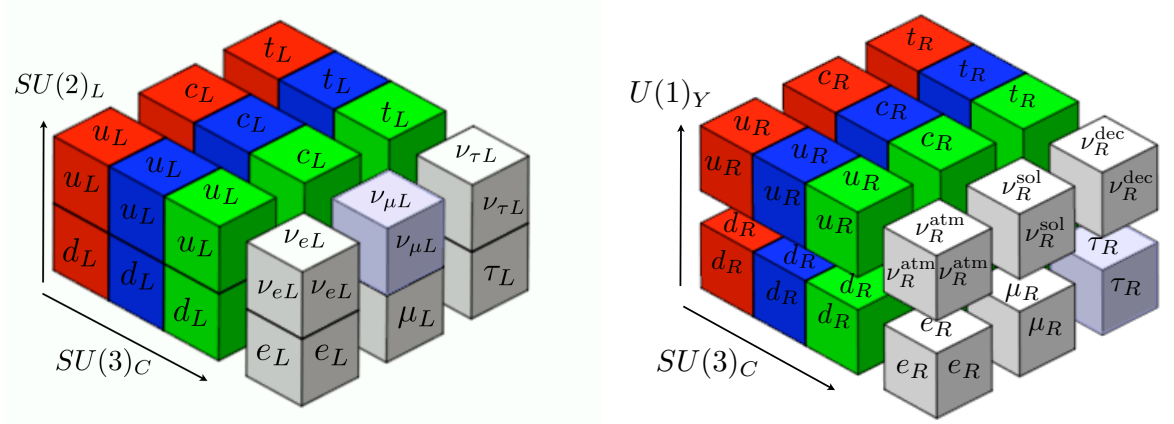

Figure 6: The Standard Model with three right-handed neutrinos defined as $\left(v_{R}^{\mathrm{atm}}, v_{R}^{\mathrm{sol}}, v_{R}^{\mathrm{dec}}\right)$ which in sequential dominance are mainly responsible for the $m_{3}, m_{2}, m_{1}$ physical neutrino masses, respectively.

trino $v_{R}^{\text {dec }}$ is approximately decoupled from the see-saw mechanism. The SM with three such right-handed neutrinos is depicted in Fig.6.

The observed pattern of lepton mixing angles can be understood in the above SD framework as follows. In the diagonal charged lepton and right-handed neutrino mass basis, if the dominant "atmospheric" right-handed neutrino has couplings $\left(m_{\mathrm{atm}}^{D}\right)^{T}=\left(0, a_{1}, a_{2}\right)$ to $\left(v_{e}, v_{\mu}, v_{\tau}\right)$, then this implies $\tan \theta_{23} \sim a_{1} / a_{2}$ [13] and a bound $\theta_{13} \lesssim m_{2} / m_{3}$ [14]. This result shows that $S D$ allows for large values of the reactor angle, consistent with the measured value. The subdominant "solar" right-handed neutrino couplings $\left(m_{\mathrm{sol}}^{D}\right)^{T}=\left(b_{1}, b_{2}, b_{3}\right)$ to $\left(v_{e}, v_{\mu}, v_{\tau}\right)$ further yield $\tan \theta_{12} \sim \sqrt{2} b_{1} /\left(b_{2}-b_{3}\right)[13,14]$. The lepton mixing angles are of course insensitive to the "decoupled" right-handed neutrino couplings $\left(m_{\mathrm{dec}}^{D}\right)^{T}$. 


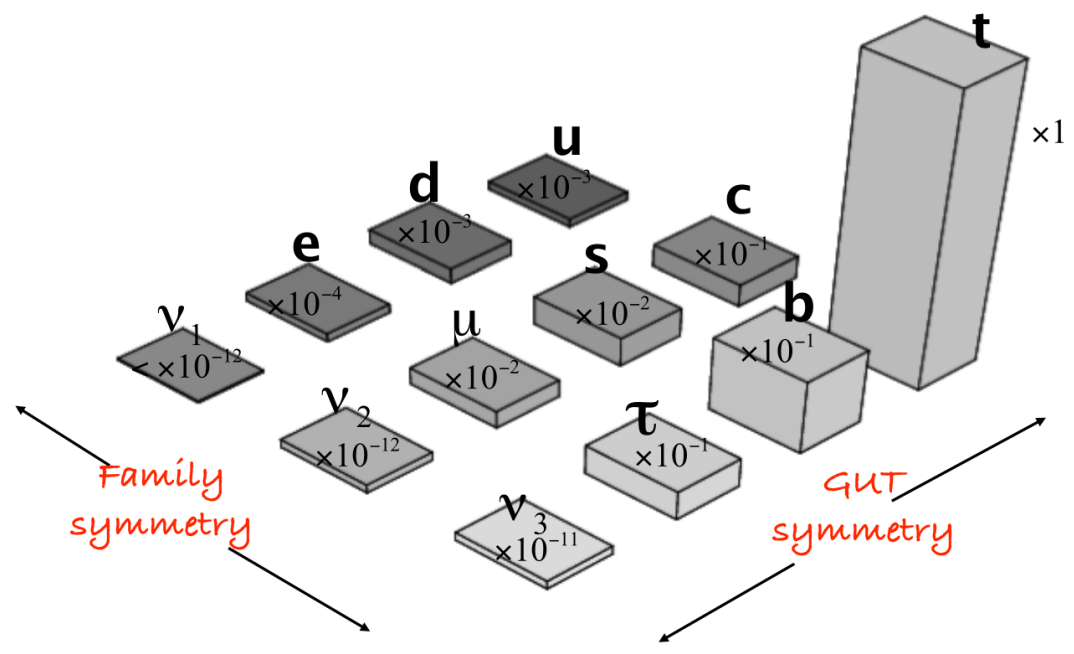

Figure 7: Quark and lepton masses lego plot (true heights need to be scaled by the factors shown).

\section{Towards a theory of flavour}

\subsection{GUTs and Family Symmetry}

If we wish to understand the pattern of quark and lepton masses and mixing angles (and $\mathrm{CP}$ violation) then a fruitful approach is to introduce GUT and family symmetry acting in the directions shown in Fig.7. Some popular family symmetries which admit triplet representations are shown in Fig.8. Some possible candidate unified gauge groups are shown in Fig. 9.

\subsection{Klein symmetry}

The starting point for family symmetry models is to consider the Klein symmetry of the neutrino mass matrix. First consider the phase symmetry of the diagonal charged lepton mass matrix $M_{e}$,

$$
T^{\dagger}\left(M_{e}^{\dagger} M_{e}\right) T=M_{e}^{\dagger} M_{e}
$$

where $T=\operatorname{diag}\left(1, \omega, \omega^{2}\right)$ and $\omega=e^{2 \pi / n}$. For example for $n=3$ clearly $T$ generates the group $Z_{3}^{T}$. In any case, the Klein symmetry of the neutrino mass matrix is given by,

$$
m^{v}=S^{T} m^{v} S, \quad m^{v}=U^{T} m^{v} U
$$

where [15]

$$
\begin{aligned}
S & =U_{\mathrm{PMNS}}^{*} \operatorname{diag}(+1,-1,-1) U_{\mathrm{PMNS}}^{T} \\
U & =U_{\mathrm{PMNS}}^{*} \operatorname{diag}(-1,+1,-1) U_{\mathrm{PMNS}}^{T} \\
S U & =U_{\mathrm{PMNS}}^{*} \operatorname{diag}(-1,-1,+1) U_{\mathrm{PMNS}}^{T}
\end{aligned}
$$




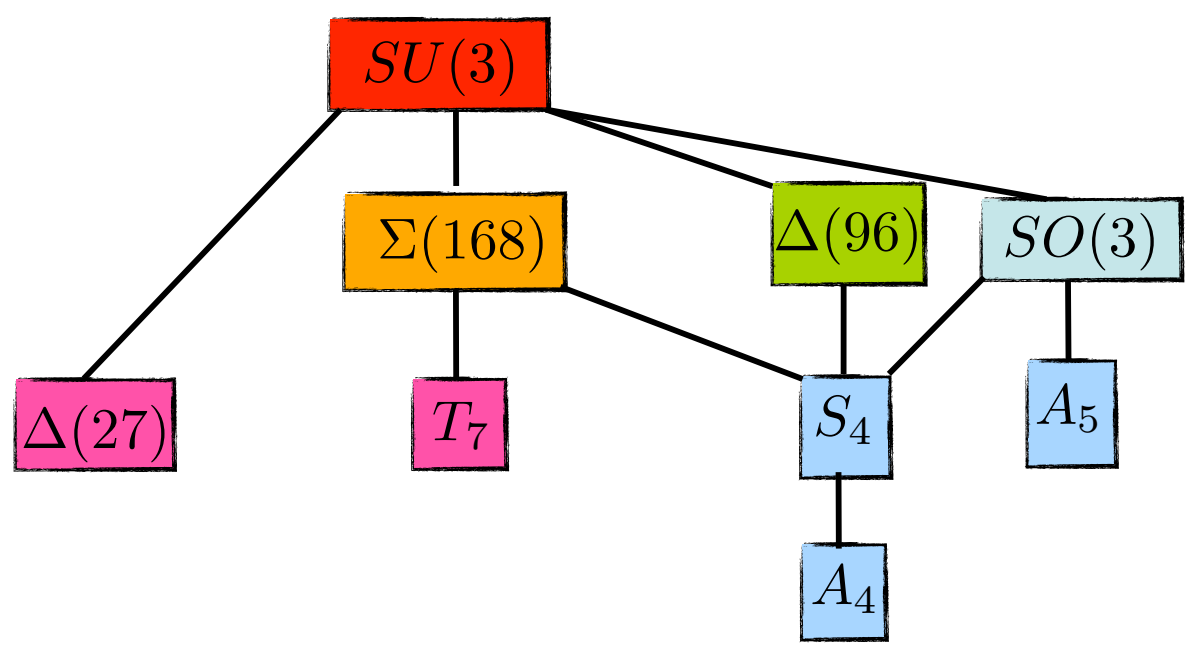

Figure 8: Some popular family symmetries and their relationships.

and

$$
\mathscr{K}=\{1, S, U, S U\}
$$

is called the Klein symmetry $Z_{2}^{S} \times Z_{2}^{U}$.

\subsection{Direct models and simple lepton mixing patterns}

The idea of direct models is that the three generators $S, T, U$ introduced above are embedded into a discrete family symmetry $G$ which is broken by new Higgs fields called "flavons" of two types: $\phi^{l}$ whose VEVs preserve $T$ and $\phi^{v}$ whose VEVs preserve $S, U$. These flavons are segregated such that $\phi^{l}$ only appears in the charged lepton sector and $\phi^{v}$ only appears in the neutrino sector as depicted in Fig.10, thereby enforcing the symmetries of the mass matrices. Note that the full Klein symmetry $Z_{2}^{S} \times Z_{2}^{U}$ of the neutrino mass matrix is enforced by symmetry in the direct approach. The Direct approach typically leads to simple patterns of mixing, as discussed below.

An early suggested pattern of lepton mixing is known as bimaximal (BM) mixing with $s_{13}^{2}=0$ and $s_{12}^{2}=s_{23}^{2}=1 / 2$ which could originate from the discrete group $S_{4}$, where the $T$ generator is preserved in the charged lepton sector, and the $S, U$ generators are preserved in the neutrino sector (identified with the Klein symmetry) as shown in Fig.10. It has a maximal solar mixing angle [16], and is given by a matrix of the form

$$
U_{\mathrm{BM}}=\left(\begin{array}{ccc}
\frac{1}{\sqrt{2}} & \frac{1}{\sqrt{2}} & 0 \\
-\frac{1}{2} & \frac{1}{2} & \frac{1}{\sqrt{2}} \\
\frac{1}{2} & -\frac{1}{2} & \frac{1}{\sqrt{2}}
\end{array}\right) .
$$

A second pattern of lepton mixing which came to dominate the model building community until the measurement of the reactor angle is the tribimaximal (TB) mixing matrix [17]. This 


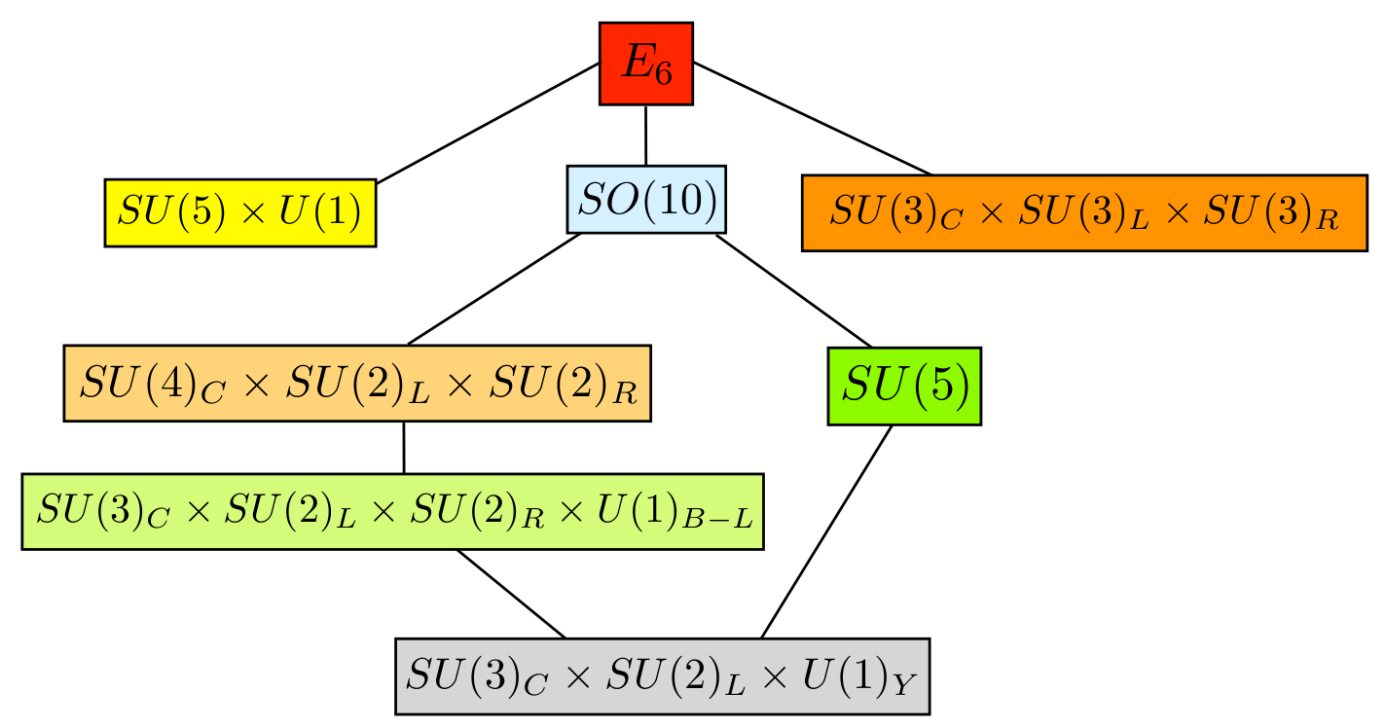

Figure 9: Some possible candidate unified gauge groups.

has been associated with models based on the flavour symmetries $\mathrm{A}_{4}$ and $\mathrm{S}_{4}$. Like BM mixing it predicts $s_{13}^{2}=0$ and $s_{23}^{2}=1 / 2$ but differs in that it predicts a solar mixing angle given by $s_{12}=$ $1 / \sqrt{3}$, i.e. $\theta_{12} \approx 35.3^{\circ}$. The mixing matrix is given explicitly by

$$
U_{\mathrm{TB}}=\left(\begin{array}{ccc}
\sqrt{\frac{2}{3}} & \frac{1}{\sqrt{3}} & 0 \\
-\frac{1}{\sqrt{6}} & \frac{1}{\sqrt{3}} & \frac{1}{\sqrt{2}} \\
\frac{1}{\sqrt{6}} & -\frac{1}{\sqrt{3}} & \frac{1}{\sqrt{2}}
\end{array}\right) .
$$

Another pattern of lepton mixing which was viable until the reactor angle measurement associates the golden ratio $\varphi=\frac{1+\sqrt{5}}{2}$ with the solar mixing angle. The original golden ratio (GR) mixing pattern is related to the flavour symmetry $A_{5}$ [18]. As above, it predicts $s_{13}^{2}=0$ and $s_{23}^{2}=1 / 2$ but differs by having a solar mixing angle given by $t_{12}^{v}=1 / \varphi$, i.e. $\theta_{12}^{v} \approx 31.7^{\circ}$, resulting in the mixing matrix

$$
U_{\mathrm{GR}}=\left(\begin{array}{ccc}
\frac{\varphi}{\sqrt{2+\varphi}} & \frac{1}{\sqrt{2+\varphi}} & 0 \\
-\frac{1}{\sqrt{4+2 \varphi}} & \frac{\varphi}{\sqrt{4+2 \varphi}} & \frac{1}{\sqrt{2}} \\
\frac{1}{\sqrt{4+2 \varphi}} & -\frac{\varphi}{\sqrt{4+2 \varphi}} & \frac{1}{\sqrt{2}}
\end{array}\right) .
$$

Following the measurement of the reactor angle, all of the above simple patterns are excluded and it has emerged that the only viable direct models are those based on $\Delta\left(6 N^{2}\right)$ which leads to more complicated patterns of lepton mixing consistent with data [19, 20, 21]. Unfortunately large $N$ is required in order to achieve the desired reactor angle. Moreover such models generally predict 


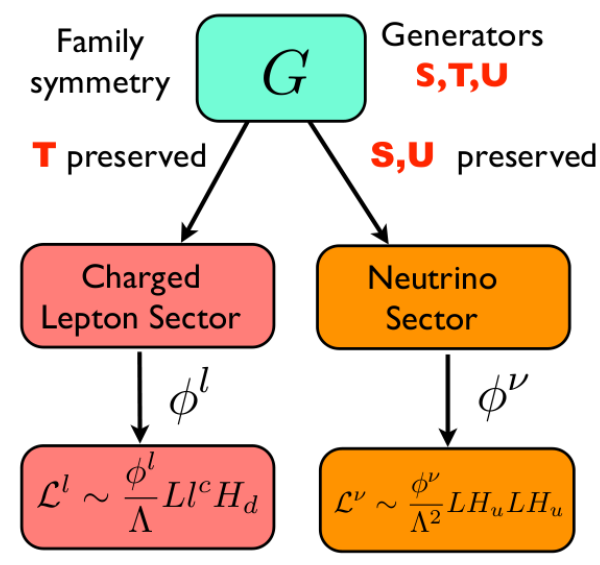

Figure 10: The direct approach to models of lepton mixing.

the CP phase $\delta=0, \pi$ resulting in the atmospheric sum rule [20],

$$
\theta_{23}=45^{\circ} \mp \theta_{13} / \sqrt{2} \text {. }
$$

which follows since the PMNS matrix has the TM2 form shown later in Eq.5.4.

\subsection{Spontaneous CP violation}

The direct approach can be generalised to the case of a conserved CP (see [22] and references therein) which is spontaneously broken as shown in Fig.11. This approach has been studied for $\Delta\left(6 N^{2}\right)$ in [23]. However since we already know that $\delta=0, \pi$ in this case it only fixes the Majorana phases.

The generalised CP approach has also been used in the semi-direct approach (defined below) where the phase $\delta$ is undetermined without $\mathrm{CP}$. Here the results are more interesting since for the smaller groups like $A_{4}$ and $S_{4}$ one generally predicts a discrete choice including $\delta= \pm \pi / 2$ [24]. However for larger groups in the series $\Delta\left(6 N^{2}\right)$ and $\Delta\left(3 N^{2}\right)$, broken in a semi-direct way, the discrete predictions for $\delta$ proliferate [25].

Recently an invariant approach to CP symmetry in family symmetry models has been discussed [26].

\section{Semi-direct approach and mixing sum rules}

Taking a less constrained approach to model building one may suppose that we start from only smaller discrete family groups such as $S_{4}$, which leads to either TB or BM mixing at leading order, or $A_{5}$ which leads to GR mixing at leading order as shown in Fig.12. Then we suppose that at higher order, one or more of the generators $S, T, U$ is broken, which is necessary in this approach since the resulting BM, TB and GR mixing patterns discussed in Eqs.4.7,4.8,4.9 are excluded. There are two interesting possibilities depicted in Fig.12 as follows:

1. If the $U$ generator is broken then this leads to either TM1 or TM2 mixing depending on whether $S U$ or $S$ is preserved, leading to atmospheric sum rules, as discussed below. 


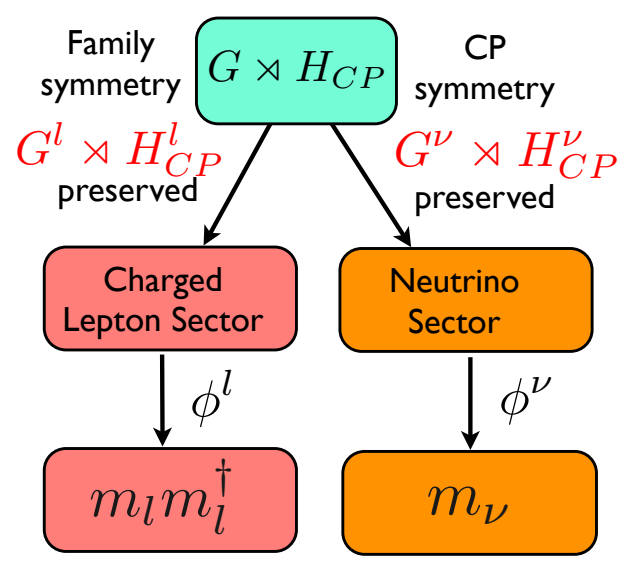

Figure 11: The direct approach including CP.

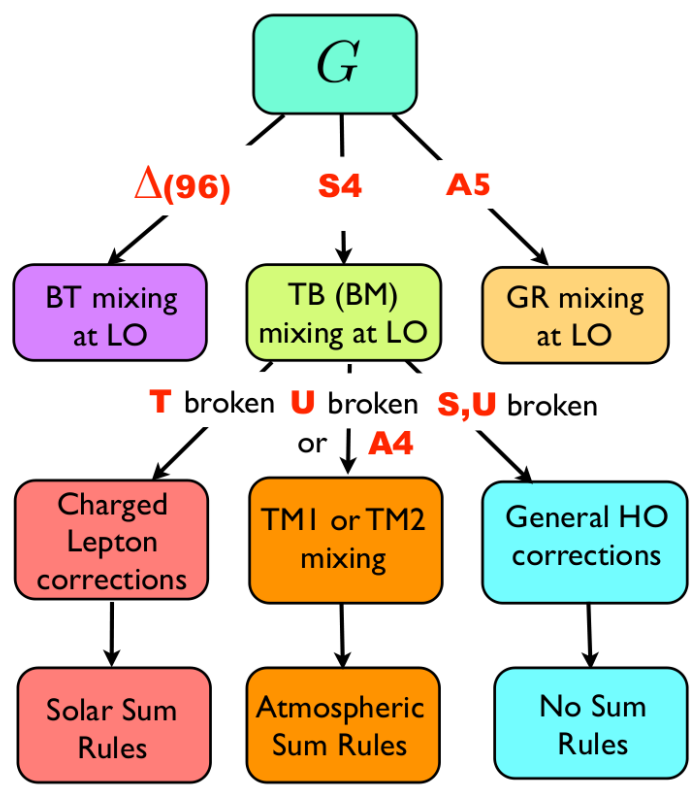

Figure 12: The semi-direct approach to models of lepton mixing.

2. If the $T$ generator protecting the charged lepton mass matrix is broken, then we can expect charged lepton corrections leading to the solar sum rules also as discussed below.

The semi-direct approach was first used in $[27,28]$ for $A_{4}$ where there is no $U$ generator to start with and also $S_{4}$ which is broken to $A_{4}$ at higher order [28]. It was subsequently generalised to von Dyck groups in [29]. In all cases the reactor angle is not predicted but described by a free parameter. This is a retreat from the original goal of predicting lepton mixing angles using symmetry.

\subsection{TB deviation parameters and atmospheric sum rules}

After the measurement of the reactor angle, TB mixing is excluded. However, TB mixing still remains a reasonable approximation to lepton mixing for the solar and atmospheric angles. It 
therefore makes sense to expand the angles about their TB values [30, 31]:

$$
\begin{aligned}
\sin \theta_{12} & =\frac{1}{\sqrt{3}}(1+s), \\
\sin \theta_{23} & =\frac{1}{\sqrt{2}}(1+a), \\
\sin \theta_{13} & =\frac{r}{\sqrt{2}},
\end{aligned}
$$

where $s, a$, and $r$ are the $(s)$ olar, $(a)$ tmospheric and $(r)$ eactor deviation parameters such that TB mixing [17] is recovered for $s=a=r=0$. For example, TBC mixing [5] corresponds to $s=a=0$ and $r=\theta_{C}$, where $\theta_{C}$ is the Cabibbo angle, which is consistent with data at three sigma as shown in Fig.3.

Tri-maximal mixing is a variation which preserves either the first or the second column of the TB mixing mixing matrix in Eq.4.8, leading to two versions called TM1 or TM2,

$$
U_{\mathrm{TM} 1}=\left(\begin{array}{cc}
\frac{2}{\sqrt{3}} & -- \\
-\frac{1}{\sqrt{6}} & -- \\
\frac{1}{\sqrt{6}} & --
\end{array}\right), U_{\mathrm{TM} 2}=\left(\begin{array}{ccc}
-\frac{1}{\sqrt{3}} & - \\
-\frac{1}{\sqrt{3}} & - \\
- & -\frac{1}{\sqrt{3}} & -
\end{array}\right),
$$

where TM1 preserves the product $S U$ of $S_{4}$, while TM2 preserves the $S$ generator of $S_{4}$. The dashes indicate that the other elements are undetermined. However these are fixed once the reactor angle is specified (it is a free parameter here). These imply the relations

$$
\begin{array}{r}
\text { TM1 : }\left|U_{e 1}\right|=\sqrt{\frac{2}{3}} \text { and }\left|U_{\mu 1}\right|=\left|U_{\tau 1}\right|=\frac{1}{\sqrt{6}} ; \\
\text { TM2: }\left|U_{e 2}\right|=\left|U_{\mu 2}\right|=\left|U_{\tau 2}\right|=\frac{1}{\sqrt{3}} .
\end{array}
$$

The atmospheric mixing sum rule

$$
a=\lambda r \cos \delta+\mathscr{O}\left(a^{2}, r^{2}\right), \text { with } s=\mathscr{O}\left(a^{2}, r^{2}\right),
$$

was first derived in [30] by expanding the PMNS matrix to first order in $r, s, a$. It also follows from a first order expansion of Eqs.5.5,5.6, where $\lambda=1$ for TM1 and $\lambda=-1 / 2$ for TM2. The study of correlations of this type, and their application to the discrimination between underlying models, has been shown to be a realistic aim for a next-generation superbeam experiment [32], see for example Fig.13.

\subsection{Charged lepton corrections and solar sum rules}

Now suppose that neutrino mixing $U_{\mathrm{TB}}^{v}$ obeys TB exactly so that the PMNS matrix is given by $U=U^{e} U_{\mathrm{TB}}^{v}$ where $U_{\mathrm{TB}}^{v}$ is equated to Eq.4.8 while $U^{e}$ encodes some unknown charged lepton corrections which must be small since $U$ is not far from TB mixing. Charged lepton corrections involve some violation of the $T$ generator.

The solar mixing sum rule $[33,34,35]$ then follows from the assumption that $\theta_{23}^{e}=\theta_{13}^{e}=0$,

$$
\theta_{12} \approx 35.26^{\circ}+\theta_{13} \cos \delta \quad \text { or } \quad \cos \delta \approx \frac{\theta_{12}-35.26^{\circ}}{\theta_{13}}
$$


where $35.26^{\circ}=\sin ^{-1} \frac{1}{\sqrt{3}}$, which can be recast as [30],

$$
s=r \cos \delta+\mathscr{O}\left(a^{2}, r^{2}\right)
$$

Recently it has been realised that, assuming only $\theta_{13}^{e}=0$, but allowing $\theta_{23}^{e} \neq 0$, the following exact result can be simply obtained [36]:

$$
\frac{\left|U_{\tau 1}\right|}{\left|U_{\tau 2}\right|}=\frac{1}{\sqrt{2}}
$$

which readily leads to an exact prediction for $\cos \delta$ in terms of the other physical lepton angles,

$$
\cos \delta=\frac{t_{23} s_{12}^{2}+s_{13}^{2} c_{12}^{2} / t_{23}-\frac{1}{3}\left(t_{23}+s_{13}^{2} / t_{23}\right)}{\sin 2 \theta_{12} s_{13}}
$$

as displayed in Fig.14. For the case of TB neutrino mixing the error in $\cos \delta$ incurred by using the leading order sum rule is $\Delta(\cos \delta) \sim 0.08$ for normal ordering and $\Delta(\cos \delta) \sim 0.01$ for inverted ordering [36]. These errors are unimportant for current levels of accuracy as shown in Fig.14 but they may (hopefully) become significant in the future.

The leading order sum rule offers a simple way to understand the results in Fig.14. For example from Fig.14 it seems that TB neutrino mixing predicts $\cos \delta \approx 0$ if $\theta_{12} \approx 35.26^{\circ}$, which is obvious from Eq.5.8 ${ }^{3}$. This can also be understood from Eq.5.11 where we see that for $s_{12}^{2}=1 / 3$ the leading terms $t_{23} s_{12}^{2}$ and $\frac{1}{3} t_{23}$ in the numerator cancel, leaving $\cos \delta=s_{13} /\left(2 \sqrt{2} t_{23}\right) \approx 0.05$ which is consistent with the numerical estimates of the error given above for a range of $\theta_{12}$.

Solar sum rules can also be obtained for different types of neutrino mixing such as $U_{\mathrm{BM}}^{v}$ (which is almost excluded) and $U_{\mathrm{GR}}^{v}$ (which gives similar results to the case $U_{\mathrm{TB}}^{v}$ considered here). The general formula given in [36] is,

$$
\cos \delta=\frac{t_{23} s_{12}^{2}+s_{13}^{2} c_{12}^{2} / t_{23}-s_{12}^{v 2}\left(t_{23}+s_{13}^{2} / t_{23}\right)}{\sin 2 \theta_{12} s_{13}}
$$

where $s_{12}^{\nu 2}=\frac{1}{3}, \frac{1}{2}$ for $U_{\mathrm{TB}, \mathrm{BM}}^{v}$ and so on. The prospects for studying solar sum rules at JUNO and LBNF is discussed in [36]. A slightly more lengthy but equivalent formula to Eq.5.11 had been previously derived [37] by an alternative method involving an auxiliary phase $\phi$ without using the elegant result Eq.5.10,

$$
\cos \delta=\frac{t_{23}}{\sin 2 \theta_{12} s_{13}}\left[\cos 2 \theta_{12}^{\nu}+\left(s_{12}^{2}-c_{12}^{\nu 2}\right)\left(1-\cot ^{2} \theta_{23} s_{13}^{2}\right)\right] .
$$

We prefer the simpler form in Eq.5.12 which involves $\theta_{12}^{v}$ in only one place since it exhibits the cancellation between the terms $t_{23} s_{12}^{2}$ and $s_{12}^{\nu 2} t_{23}$ when $s_{12}^{2}=s_{12}^{\nu 2}$ responsible for the prediction $\cos \delta \approx 0$ in this case

Finally we give a word of caution that when comparing leading order sum rules to the exact results the ratio $(\cos \delta)_{\text {exact }} /(\cos \delta)_{\mathrm{LO}}$ used in [38] will lead to misleading results when $(\cos \delta)_{\mathrm{LO}} \approx$ 0 . In general it is safer to compare them using the experimentally relevant quantity $\Delta(\cos \delta)=$

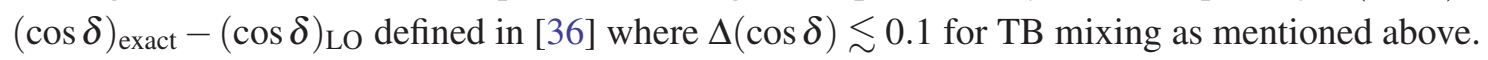




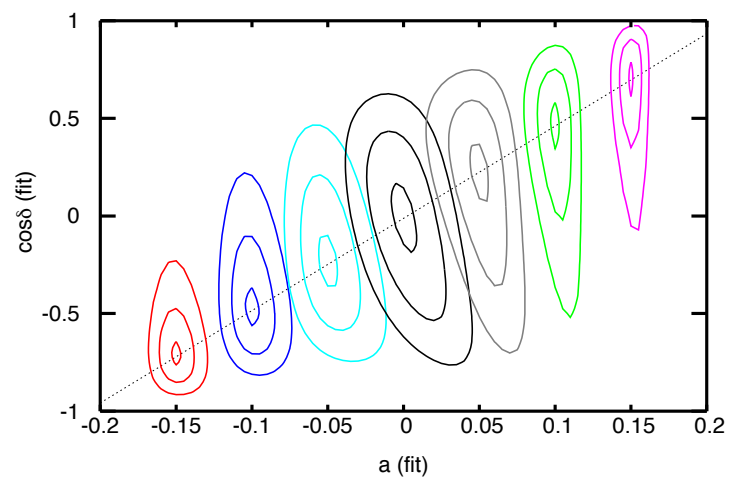

Figure 13: Expectation for the determination of the TM1 atmospheric mixing sum rule $a \approx r \cos \delta$ at one, three and five sigma for a low energy neutrino factory with a magnetised iron detector (for more details see [32]).

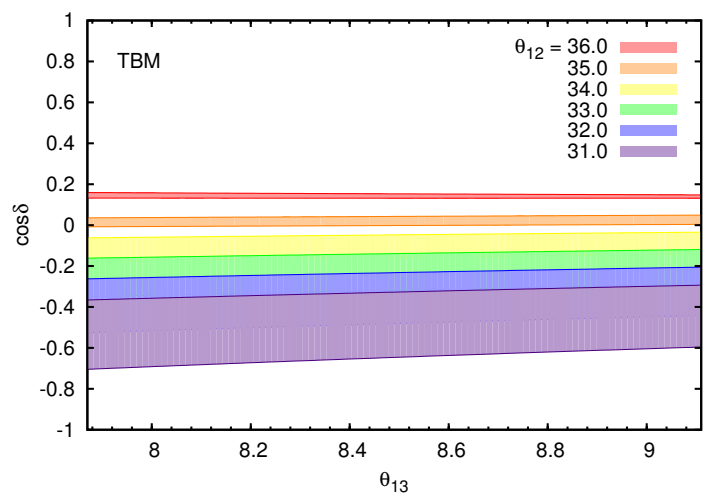

Figure 14: Solar sum rules prediction for $\cos \delta$ using the exact result for TB neutrino mixing

\section{Indirect models}

The final logical possibility is that the family symmetry is completely broken as shown in Fig.15. In this approach, we allow the flavons $\phi^{l}$ and $\phi^{v}$ to have not only symmetry preserving vacuum alignments, but also new alignments which are orthogonal to them and break the symmetry.

For example in $A_{4}$, we have the symmetry preserving alignments,

$$
\left(\begin{array}{l}
v \\
0 \\
0
\end{array}\right),\left(\begin{array}{l}
0 \\
v \\
0
\end{array}\right),\left(\begin{array}{l}
0 \\
0 \\
v
\end{array}\right),\left(\begin{array}{c} 
\pm v \\
\pm v \\
\pm v
\end{array}\right),
$$

where the first three alignments may be used to make the charged lepton mass matrix diagonal. In the neutrino sector we consider new orthogonal alignments such as,

$$
\left(\begin{array}{l}
0 \\
a \\
a
\end{array}\right) \perp\left(\begin{array}{c}
v \\
v \\
-v
\end{array}\right),\left(\begin{array}{l}
v \\
0 \\
0
\end{array}\right),
$$

\footnotetext{
${ }^{3}$ Note that $\cos \delta \sim 0$ is consistent with the current hint $\delta \sim-\pi / 2$.
} 


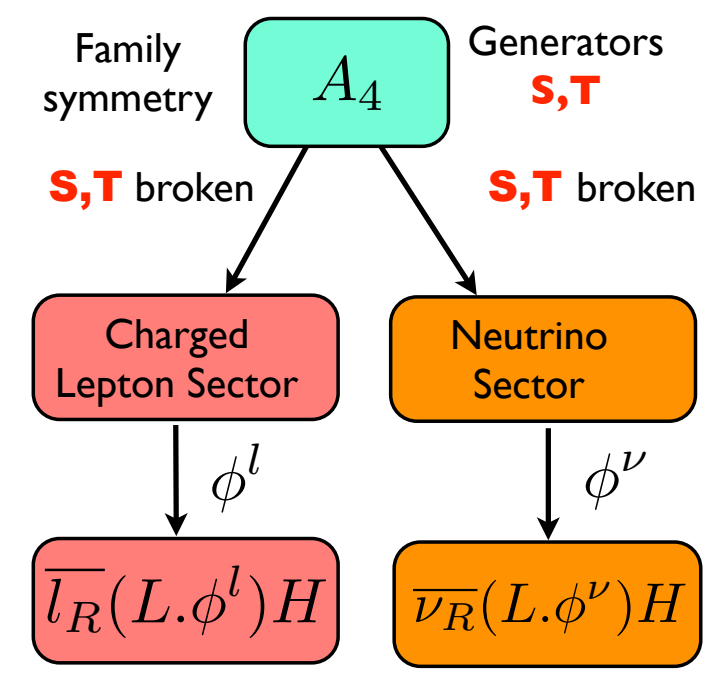

Figure 15: The indirect approach to models of lepton mixing.

as well as ones which are orthogonal to them, and so on. Such alignments completely break the $A_{4}$ symmetry. The following symmetry breaking alignment may be obtained using orthogonality arguments

$$
\left(\begin{array}{c}
2 \\
-1 \\
1
\end{array}\right) \perp\left(\begin{array}{c}
1 \\
1 \\
-1
\end{array}\right),\left(\begin{array}{l}
0 \\
1 \\
1
\end{array}\right) .
$$

Using such "orthogonal" alignments various types of constrained sequential dominance (CSD) have been proposed, with the dominant right-handed "atmospheric" couplings $(0, a, a)$ to $\left(v_{e}, v_{\mu}, v_{\tau}\right)$, provided by the alignment in Eq.6.2, and hence an approximate maximal atmospheric angle $\tan \theta_{23} \sim$ $a_{1} / a_{2} \sim 1$. Further orthogonal alignments are responsible for the subdominant "solar" right-handed neutrino couplings to $\left(v_{e}, v_{\mu}, v_{\tau}\right)$ as follows:

- $\operatorname{CSD}(1):(b, b,-b) \rightarrow \theta_{13}=0[33]$.

- $\operatorname{CSD}(2):(b, 2 b, 0) \rightarrow \theta_{13} \sim 7^{\circ}[39]$.

- $\operatorname{CSD}(3):(b, 3 b, b) \rightarrow \theta_{13} \sim 8.5^{\circ}[40]$.

- $\operatorname{CSD}(4):(b, 4 b, 2 b) \rightarrow \theta_{13} \sim 9^{\circ}[40]$.

These examples are chosen to maintain an approximate trimaximal solar angle $\tan \theta_{12} \sim \sqrt{2} b_{1} /\left(b_{2}-\right.$ $\left.b_{3}\right) \sim 1 / \sqrt{2}$ but have different reactor angles depending on the phase $\arg (b / a)$, leading to a prediction for $\delta$ in each case.

The $\operatorname{CSD}(n)$ alignment $(1, n,(n-2))^{T}$ is orthogonal to the alignment in Eq. 6.3,

$$
\left(\begin{array}{c}
1 \\
n \\
(n-2)
\end{array}\right) \perp\left(\begin{array}{c}
2 \\
-1 \\
1
\end{array}\right),
$$


where the orthogonality in Eq. 6.4 is maintained for any value of $n$ (not necessarily integer). To pin down the value of $n$ and show that it is a particular integer requires a further orthogonality condition as discussed in [42].

In a $\operatorname{CSD}(n)$ framework [42], after the see-saw mechanism, the neutrino mass matrix simplifies in the two right-handed neutrino case to

$$
m_{(n)}^{v}=m_{a}\left(\begin{array}{lll}
0 & 0 & 0 \\
0 & 1 & 1 \\
0 & 1 & 1
\end{array}\right)+m_{b} e^{i \eta}\left(\begin{array}{ccc}
1 & n & n-2 \\
n & n^{2} & n(n-2) \\
n-2 & n(n-2) & (n-2)^{2}
\end{array}\right),
$$

where $\eta$ is a physically important phase. This case immediately predicts the lightest physical neutrino mass to be zero, $m_{1}=0$. It also immediately predicts TM1 mixing since,

$$
m_{(n)}^{v}\left(\begin{array}{c}
2 \\
-1 \\
1
\end{array}\right)=\left(\begin{array}{l}
0 \\
0 \\
0
\end{array}\right)
$$

In other words the column vector $(2,-1,1)^{T}$ is an eigenvector of $m_{(n)}^{v}$ with a zero eigenvalue, i.e. it is the first column of the PMNS mixing matrix, corresponding to $m_{1}=0$, which means TM1 mixing.

For a given choice of alignment $n$, there are three real input parameters $m_{a}, m_{b}$ and $\eta$ from which two light physical neutrino masses $m_{2}, m_{3}$, three lepton mixing angles, the CP-violating phase $\delta_{C P}$ and two Majorana phases are derived; a total of nine physical parameters from three input parameters, i.e. six predictions for each value of $n$. As the Majorana phases are not known and $\delta_{C P}$ is only tentatively constrained by experiment, this leaves five presently measured observables, namely the two neutrino mass squared differences and the three lepton mixing angles, from only three input parameters. The resulting predictions [42] are shown in Fig.16.

\subsection{A to $\mathrm{Z}$ of flavour with Pati-Salam}

As an example of an "indirect" model, an "A to Z of flavour with Pati-Salam" based on the Pati-Salam gauge group has been proposed [43] as sketched in Fig.17. The Pati-Salam symmetry leads to $Y^{u}=Y^{v}$, with the first column proportional to the orthogonal alignment $(0, e, e)$ the second column proportional to the $\operatorname{CSD}(4)$ orthogonal alignment, $(a, 4 a, 2 a)$ and the third column is proportional to the symmetry preserving alignment $(0,0, c)$, where $e \ll a \ll c$ gives the hierarchy $m_{u} \ll m_{c} \ll m_{t}$. This structure predicts a Cabibbo angle $\theta_{C} \approx 1 / 4$ in the diagonal $Y^{d} \sim Y^{e}$ basis enforced by the first three alignments in Eq.6.1. It also predicts a normal neutrino mass hierarchy with $\theta_{13} \approx 9^{\circ}, \theta_{23} \approx 45^{\circ}$ and $\delta \approx 260^{\circ}$ [43].

The model is based on the Pati-Salam (PS) gauge group, with $A_{4} \times Z_{5}$ family symmetry,

$$
S U(4)_{C} \times S U(2)_{L} \times S U(2)_{R} \times A_{4} \times Z_{5} .
$$

The quarks and leptons are unified in the PS representations as follows,

$$
\begin{aligned}
& F_{i}=(4,2,1)_{i}=\left(\begin{array}{llll}
u & u & u & v \\
d & d & d & e
\end{array}\right)_{i} \rightarrow\left(Q_{i}, L_{i}\right), \\
& F_{i}^{c}=(\overline{4}, 1,2)_{i}=\left(\begin{array}{llll}
u^{c} & u^{c} & u^{c} & v^{c} \\
d^{c} & d^{c} & d^{c} & e^{c}
\end{array}\right)_{i} \rightarrow\left(u_{i}^{c}, d_{i}^{c}, v_{i}^{c}, e_{i}^{c}\right),
\end{aligned}
$$



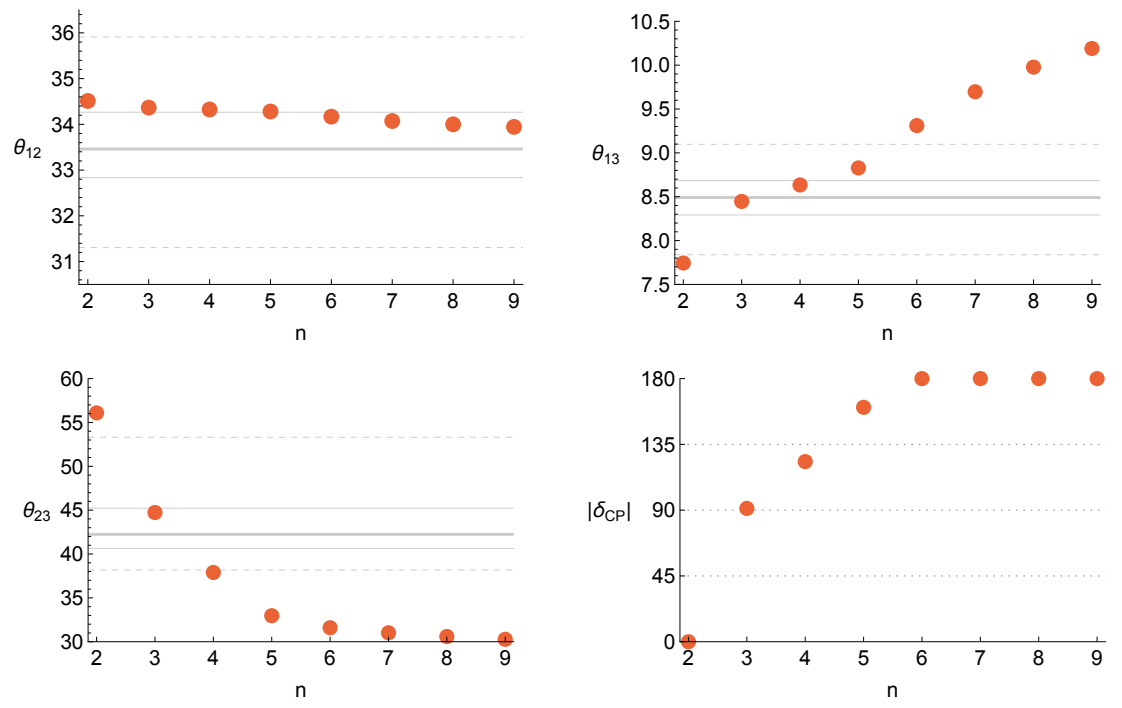

Figure 16: Best-fit PMNS mixing angles and CP-violating phase with respect to $n$, for the two right-handed neutrino $\operatorname{CSD}(n)$ model, after performing a global fit to the three real input parameters $m_{a}, m_{b}$ and $\eta$. We emphasise that $\left|\delta_{\mathrm{CP}}\right|$ is a genuine prediction here since have not used the one sigma hint from experiment as an input constraint. It is striking that both $\operatorname{CSD}(3)$ and $\operatorname{CSD}(4)$ both yield predictions within the preferred range $\left|\delta_{\mathrm{CP}}\right| \sim 90^{\circ} \pm 45^{\circ}$ but may be distinguished by their differing predictions for the atmospheric angle $\theta_{23} \approx 45^{\circ}$ and $\theta_{23} \approx 38^{\circ}$, respectively.
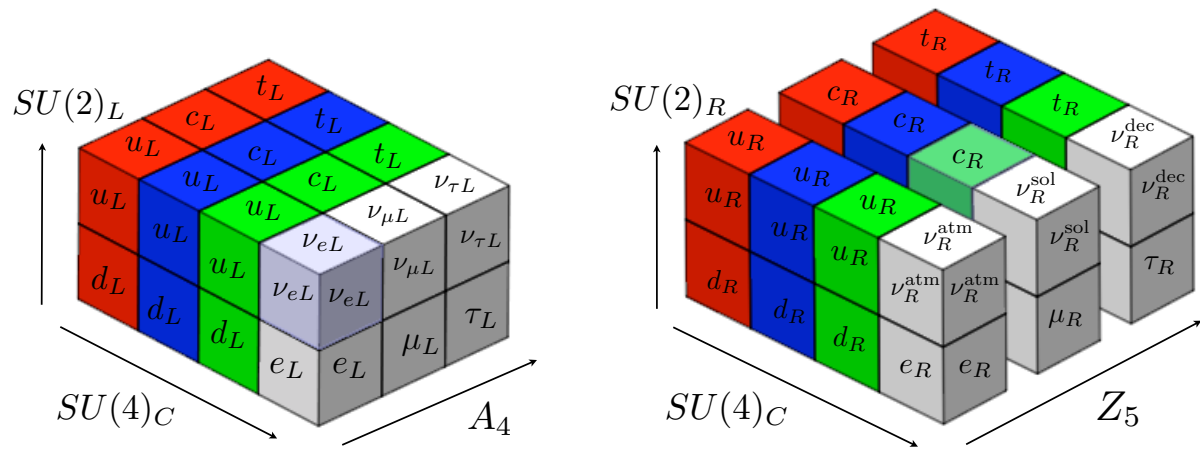

Figure 17: $A$ to $Z$ of flavour with Pati-Salam, where $A \equiv A_{4}$ and $Z \equiv Z_{5}$. The left-handed families form a triplet of $A_{4}$ and are doublets of $S U(2)_{L}$. The right-handed families are distinguished by $Z_{5}$ and are doublets of $S U(2)_{R}$. The $S U(4)_{C}$ unifies the quarks and leptons with leptons as the fourth colour, depicted here as white.

where the SM multiplets $Q_{i}, L_{i}, u_{i}^{c}, d_{i}^{c}, v_{i}^{c}, e_{i}^{c}$ resulting from PS breaking are also shown and the subscript $i(=1,2,3)$ denotes the family index. The left-handed quarks and leptons form an $A_{4}$ triplet $F$, while the three (CP conjugated) right-handed fields $F_{i}^{c}$ are $A_{4}$ singlets, distinguished by $Z_{5}$ charges $\alpha, \alpha^{3}, 1$, for $i=1,2,3$, respectively. Clearly the Pati-Salam model cannot be embedded into an $S O(10)$ Grand Unified Theory (GUT) since different components of the 16-dimensional representation of $S O(10)$ would have to transform differently under $A_{4} \times Z_{5}$, which is impossible. On the other hand, the PS gauge group and $A_{4}$ could emerge directly from string theory.

The Pati-Salam gauge group is broken at the GUT scale to the SM,

$$
S U(4)_{C} \times S U(2)_{L} \times S U(2)_{R} \rightarrow S U(3)_{C} \times S U(2)_{L} \times U(1)_{Y}
$$


by PS Higgs, $H^{c}$ and $\overline{H^{c}}$,

$$
\begin{aligned}
& H^{c}=(\overline{4}, 1,2)=\left(u_{H}^{c}, d_{H}^{c}, v_{H}^{c}, e_{H}^{c}\right), \\
& \overline{H^{c}}=(4,1,2)=\left(\bar{u}_{H}^{c}, \bar{d}_{H}^{c}, \bar{v}_{H}^{c}, \bar{e}_{H}^{c}\right) .
\end{aligned}
$$

These acquire vacuum expectation values (VEVs) in the "right-handed neutrino" directions, with equal VEVs close to the GUT scale $2 \times 10^{16} \mathrm{GeV}$,

$$
\left\langle H^{c}\right\rangle=\left\langle v_{H}^{c}\right\rangle=\left\langle\overline{H^{c}}\right\rangle=\left\langle\bar{v}_{H}^{c}\right\rangle \sim 2 \times 10^{16} \mathrm{GeV},
$$

so as to maintain supersymmetric gauge coupling unification.

Our starting point is to assume that the high energy theory, above the PS breaking scale, conserves CP. Under a CP transformation, the $A_{4}$ singlet fields $\xi, \Sigma_{u}, \Sigma_{d}$ transform into their complex conjugates,

$$
\xi \rightarrow \xi^{*}, \Sigma_{u} \rightarrow \Sigma_{u}^{*}, \Sigma_{d} \rightarrow \Sigma_{d}^{*},
$$

where the complex conjugate fields transform in the complex conjugate representations under $A_{4} \times$ $Z_{5}$. For example if $\xi \sim \alpha^{4}$, under $Z_{5}$, then $\xi^{*} \sim \alpha$. Similarly if $\Sigma_{u} \sim 1^{\prime}, \Sigma_{d} \sim 1^{\prime \prime}$, under $A_{4}$, then $\Sigma_{u}^{*} \sim 1^{\prime \prime}, \Sigma_{d}^{*} \sim 1^{\prime}$. On the other hand, in a particular basis, for $A_{4}$ triplets $\phi \sim\left(\phi_{1}, \phi_{2}, \phi_{3}\right)$, a consistent definition of $\mathrm{CP}$ symmetry requires the second and third triplet components to swap under $\mathrm{CP}$,

$$
\phi \rightarrow\left(\phi_{1}^{*}, \phi_{3}^{*}, \phi_{2}^{*}\right) .
$$

With the above definition of $\mathrm{CP}$, all coupling constants $g$ and explicit masses $m$ are real due to CP conservation and the only source of phases can be the VEVs of fields which break $A_{4} \times Z_{5}$. In the model of interest, all the physically interesting CP phases will arise from $Z_{5}$ breaking.

Let us now consider the $A_{4}$ triplet fields $\phi$ which also carry $Z_{5}$ charges. In the full model there are four such triplet fields, or "flavons", denoted as $\phi_{1}^{u}, \phi_{2}^{u}, \phi_{1}^{d}, \phi_{2}^{d}$. The idea is that $\phi_{i}^{u}$ are responsible for up-type quark flavour, while $\phi_{i}^{d}$ are responsible for down-type quark flavour.

The structure of the Yukawa matrices depends on the so-called CSD(4) vacuum alignments of these flavons, with the overall phases quantised due to $Z_{5}$,

$$
\left\langle\phi_{1}^{u}\right\rangle=\frac{V_{1}^{u}}{\sqrt{2}} e^{i m \pi / 5}\left(\begin{array}{l}
0 \\
1 \\
1
\end{array}\right), \quad\left\langle\phi_{2}^{u}\right\rangle=\frac{V_{2}^{u}}{\sqrt{21}} e^{i m \pi / 5}\left(\begin{array}{l}
1 \\
4 \\
2
\end{array}\right)
$$

and

$$
\left\langle\phi_{1}^{d}\right\rangle=V_{1}^{d} e^{i n \pi / 5}\left(\begin{array}{l}
1 \\
0 \\
0
\end{array}\right), \quad\left\langle\phi_{2}^{d}\right\rangle=V_{2}^{d} e^{i n \pi / 5}\left(\begin{array}{l}
0 \\
1 \\
0
\end{array}\right) .
$$

We note here that the vacuum alignments in Eq.6.15 and the first alignment in Eq.6.14 are fairly "standard" alignments that are encountered in tri-bimaximal mixing models, while the second alignment in Eq.6.14 is obtained using orthogonality arguments, as discussed above.

The model will involve Higgs bi-doublets of two kinds, $h_{u}$ which lead to up-type quark and neutrino Yukawa couplings and $h_{d}$ which lead to down-type quark and charged lepton Yukawa 
couplings. In addition a Higgs bidoublet $h_{3}$, which is also an $A_{4}$ triplet, is used to give the third family Yukawa couplings.

After the PS and $A_{4}$ breaking, most of these Higgs bi-doublets will get high scale masses and will not appear in the low energy spectrum. In fact only two light Higgs doublets will survive down to the TeV scale, namely $H_{u}$ and $H_{d}$. The basic idea is that the light Higgs doublet $H_{u}$ with hypercharge $Y=+1 / 2$, which couples to up-type quarks and neutrinos, is a linear combination of components of the Higgs bi-doublets of the kind $h_{u}$ and $h_{3}$, while the light Higgs doublet $H_{d}$ with hypercharge $Y=-1 / 2$, which couples to down-type quarks and charged leptons, is a linear combination of components of Higgs bi-doublets of the kind $h_{d}$ and $h_{3}$,

$$
h_{u}, h_{3} \rightarrow H_{u}, \quad h_{d}, h_{3} \rightarrow H_{d}
$$

The renormalisable Yukawa operators, which respect PS and $A_{4}$ symmetries, have the following form, leading to the third family Yukawa couplings shown, using Eqs.6.8,6.16,

$$
F . h_{3} F_{3}^{c} \rightarrow Q_{3} H_{u} u_{3}^{c}+Q_{3} H_{d} d_{3}^{c}+L_{3} H_{u} v_{3}^{c}+L_{3} H_{d} e_{3}^{c},
$$

where we have used Eqs.6.8,6.16. The non-renormalisable operators, which respect PS and $A_{4}$ symmetries, have the following form,

$$
\begin{aligned}
& F . \phi_{i}^{u} h_{u} F_{i}^{c} \rightarrow Q .\left\langle\phi_{i}^{u}\right\rangle H_{u} u_{i}^{c}+L .\left\langle\phi_{i}^{u}\right\rangle H_{u} v_{i}^{c}, \\
& F . \phi_{i}^{d} h_{d} F_{i}^{c} \rightarrow Q .\left\langle\phi_{i}^{d}\right\rangle H_{d} d_{i}^{c}+L .\left\langle\phi_{i}^{d}\right\rangle H_{d} e_{i}^{c},
\end{aligned}
$$

where $i=1$ gives the first column of each Yukawa matrix, while $i=2$ gives the second column and we have used Eqs.6.8,6.16. Thus the third family masses are naturally larger since they correspond to renormalisable operators, while the hierarchy between first and second families arises from a hierarchy of flavon VEVs.

Inserting the vacuum alignments in Eqs.6.14 and 6.15 into Eqs.6.18 and 6.19, together with the renormalisable third family couplings in Eq.6.17, gives the Yukawa matrices of the form,

$$
Y^{u}=Y^{v}=\left(\begin{array}{ccc}
0 & a & 0 \\
e & 4 a & 0 \\
e & 2 a & c
\end{array}\right), \quad Y^{d} \sim Y^{e} \sim\left(\begin{array}{ccc}
y_{d}^{0} & 0 & 0 \\
0 & y_{s}^{0} & 0 \\
0 & 0 & y_{b}^{0}
\end{array}\right) .
$$

The PS unification predicts the equality of Yukawa matrices $Y^{u}=Y^{v}$ and $Y^{d} \sim Y^{e}$, while the $A_{4}$ vacuum alignment predicts the structure of each Yukawa matrix, essentially identifying the first two columns with the vacuum alignments in Eqs.6.14 and 6.15. With a diagonal right-handed Majorana mass matrix, $Y^{v}$ leads to a successful prediction of the PMNS mixing parameters. Also the Cabibbo angle is given by $\theta_{C} \approx 1 / 4$ [41]. Thus Eq.6.20 is a good starting point for a theory of quark and lepton masses and mixing, although the other quark mixing angles and the quark $\mathrm{CP}$ phase are approximately zero. However the above discussion ignores the effect of Clebsch factors which will alter the relationship between elements of $Y^{d}$ and $Y^{e}$, which also include off-diagonal elements responsible for small quark mixing angles in the full model discussed in [43]. 


\section{F-theory origin of discrete family symmetry}

F-theory models have attracted considerable interest over the recent years [44]. For example, Supersymmetric (SUSY) Grand Unified Theories (GUTs) based on $S U(5)$ has been shown to emerge naturally from F-theory. However, in the F-theory context, the $S U(5)$ GUT group is only one part of a larger symmetry. The other parts manifest themselves at low energies as Abelian and/or non-Abelian discrete symmetries, which can be identified as family symmetries, leading to significant constraints in the effective superpotential (for a review see e.g. [45]).

In [46] non-Abelian fluxes were conjectured to give rise to non-Abelian discrete family symmetries in the low energy effective theory. The origin of such a symmetry is the non-Abelian $S U(5)_{\perp}$ which accompanies $S U(5)_{G U T}$ at the $E_{8}$ point of enhancement. Whether a non-Abelian symmetry survives in the low energy theory will depend on the geometry of the compactified space and the fluxes present. The usual assumption is that the $S U(5)_{\perp}$ is first broken to a product of $U(1)_{\perp}$ groups which are then further broken by the action of discrete symmetries associated with the monodromy group. Instead it was conjectured in [46] that non-Abelian fluxes can break $S U(5)_{\perp}$ first to a non-Abelian discrete group $S_{4}$ then to a smaller group such as $A_{4}, D_{4}$ and so on which act as a family symmetry group in the low energy effective theory. Models of this kind have recently been discussed in [47]. Thus F-theory could provide the origin of the discrete family symmetries discussed in this talk.

\section{Conclusion}

In conclusion, although the reactor angle has been accurately measured, which rules out simple patterns of lepton mixing such as BM, TB and GR, it is still possible to have simple patterns of lepton mixing with the first or second column of the TB matrix preserved, namely TM1 or TM2, with atmospheric sum rules. It is also possible to maintain BM, TB and GR mixing for neutrinos with the reactor angle is due to charged lepton corrections, leading to solar sum rules.

Although adding right-handed neutrinos is a very simple and minimal thing to do, the number of right-handed (sterile) neutrinos is undetermined by anomaly cancellation, and their mass spectrum is completely unknown. The classic see-saw mechanism would correspond to having three right-handed neutrinos with masses in the range TeV-M $\mathrm{MUT}_{\mathrm{GUT}}$. Sequential dominance provides an elegant and natural way to understand neutrino mixing angles, with the dominant right-handed neutrino couplings providing the atmospheric mixing angle, the sub-dominant solar right-handed neutrino couplings providing the solar mixing angle and the decoupled right-handed neutrino couplings being irrelevant.

Turning to theories of flavour, a very promising approach is the combination of GUT and family symmetry. The large lepton mixing angles suggest some sort of discrete family symmetry at work, although not in the most simple direct way imagined before the reactor angle was measured. However the direct symmetry approach in which the symmetries of the mass matrices are directly embedded into the family symmetry, drives us to family symmetry groups in the $\Delta\left(6 N^{2}\right)$ series with large $N$ values necessary in order to explain the reactor angle.

A retreat from this is to suppose that only part of the symmetries of the mass matrices can be found in the family symmetry group, which is called the semi-direct approach. This allows smaller 
family groups such as $S_{4}, A_{4}, A_{5}$ whose generators $S, T, U$ may only partly survive. For example if $T$ is broken but the Klein symmetry $S, U$ survives in the neutrino sector this would correspond to $\mathrm{BM}, \mathrm{TB}$ or GR neutrino mixing but with charged lepton corrections, leading to solar sum rules. If $T$ is preserved but $U$ is broken then this corresponds to TM1 or TM2 mixing with atmospheric sum rules.

Alternatively, it is possible to have a small family symmetry such as $A_{4}$ where the family symmetry is completely broken, as in the indirect approach. In this case new vacuum alignments are possible which can be used to give interesting Yukawa couplings corresponding to different types of constrained sequential dominance. Although not enforced by symmetry, the indirect approach leads to highly predictive models which can be tested experimentally. We have discussed one such example, the $\mathrm{A}$ to $\mathrm{Z}$ of flavour with Pati-Salam. Finally we mentioned that the discrete family symmetries discussed in this talk might have an F-theory origin.

SK acknowledges support from the European Union FP7 ITN-INVISIBLES (Marie Curie Actions, PITN- GA-2011- 289442) and thanks the organisers for a very stimulating and enjoyable workshop.

\section{References}

[1] B. Z. Hu [Daya Bay Collaboration], arXiv:1505.03641 [hep-ex].

[2] M. C. Gonzalez-Garcia, M. Maltoni and T. Schwetz, arXiv:1409.5439.

[3] F. Capozzi, G. L. Fogli, E. Lisi, A. Marrone, D. Montanino and A. Palazzo, Phys. Rev. D 89 (2014) 093018 [arXiv:1312.2878].

[4] D. V. Forero, M. Tortola and J. W. F. Valle, arXiv:1405.7540.

[5] S. F. King, Phys. Lett. B 718 (2012) 136 [arXiv:1205.0506 [hep-ph]].

[6] S. F. King, Rept. Prog. Phys. 67 (2004) 107 [hep-ph/0310204].

[7] G. Altarelli and F. Feruglio, Rev. Mod. Phys. 82, 2701 (2010) [arXiv:1002.0211 [hep-ph]].

[8] H. Ishimori, T. Kobayashi, H. Ohki, Y. Shimizu, H. Okada and M. Tanimoto, Prog. Theor. Phys. Suppl. 183, 1 (2010) [arXiv:1003.3552 [hep-th]]; Lect. Notes Phys. 858, 1 (2012); Fortsch. Phys. 61, 441 (2013).

[9] S. F. King and C. Luhn, Rept. Prog. Phys. 76 (2013) 056201 [arXiv:1301.1340].

[10] S. F. King, A. Merle, S. Morisi, Y. Shimizu and M. Tanimoto, New J. Phys. 16 (2014) 045018 [arXiv:1402.4271].

[11] P. Minkowski, Phys. Lett. B 67 (1977) 421; M. Gell-Mann, P. Ramond and R. Slansky in Sanibel Talk, CALT-68-709, Feb 1979, and in Supergravity, North Holland, Amsterdam (1979); T. Yanagida in Proc. of the Workshop on Unified Theory and Baryon Number of the Universe, KEK, Japan (1979); S.L.Glashow, Cargese Lectures (1979); R. N. Mohapatra and G. Senjanovic, Phys. Rev. Lett. 44 (1980) 912.

[12] M. Drewes, arXiv:1502.06891 [hep-ph].

[13] S. F. King, Phys. Lett. B 439 (1998) 350 [hep-ph/9806440]; S. F. King, Nucl. Phys. B 562 (1999) 57 [hep-ph/9904210]; S. F. King, Nucl. Phys. B 576 (2000) 85 [hep-ph/9912492]. 
[14] S. F. King, JHEP 0209 (2002) 011 [hep-ph/0204360]; S. F. King, Phys. Rev. D 67 (2003) 113010 [hep-ph/0211228].

[15] S. F. King and C. Luhn, JHEP 0910 (2009) 093 [arXiv:0908.1897].

[16] M. Fukugita, M. Tanimoto and T. Yanagida, Phys. Rev. D 57 (1998) 4429 [hep-ph/9709388]; V. D. Barger, S. Pakvasa, T. J. Weiler and K. Whisnant, Phys. Lett. B 437 (1998) 107 [hep-ph/9806387]; S. Davidson and S. F. King, Phys. Lett. B 445 (1998) 191 [hep-ph/9808296].

[17] P. F. Harrison, D. H. Perkins and W. G. Scott, Phys. Lett. B 530 (2002) 167 [hep-ph/0202074].

[18] A. Datta, F. S. Ling and P. Ramond, Nucl. Phys. B 671 (2003) 383 [hep-ph/0306002]; L. L. Everett and A. J. Stuart, Phys. Rev. D 79 (2009) 085005 [arXiv:0812.1057]; F. Feruglio and A. Paris, JHEP 1103 (2011) 101 [arXiv:1101.0393].

[19] M. Holthausen, K. S. Lim and M. Lindner, Phys. Lett. B 721 (2013) 61 [arXiv:1212.2411 [hep-ph]].

[20] S. F. King, T. Neder and A. J. Stuart, Phys. Lett. B 726 (2013) 312 [arXiv:1305.3200 [hep-ph]].

[21] R. M. Fonseca and W. Grimus, JHEP 1409 (2014) 033 [arXiv:1405.3678 [hep-ph]].

[22] M. Holthausen, M. Lindner and M. A. Schmidt, JHEP 1304 (2013) 122 [arXiv:1211.6953 [hep-ph]].

[23] S. F. King and T. Neder, Phys. Lett. B 736 (2014) 308 [arXiv:1403.1758 [hep-ph]].

[24] F. Feruglio, C. Hagedorn and R. Ziegler, JHEP 1307, 027 (2013) [arXiv:1211.5560 [hep-ph]]; F. Feruglio, C. Hagedorn and R. Ziegler, Eur. Phys. J. C 74, 2753 (2014) [arXiv:1303.7178 [hep-ph]]; G. -J. Ding, S. F. King, C. Luhn and A. J. Stuart, JHEP 1305, 084 (2013) [arXiv:1303.6180 [hep-ph]]; G. -J. Ding, S. F. King and A. J. Stuart, JHEP 1312 (2013) 006 [arXiv:1307.4212].

[25] C. Hagedorn, A. Meroni and E. Molinaro, arXiv:1408.7118 [hep-ph]; G. J. Ding, S. F. King and T. Neder, JHEP 1412 (2014) 007 [arXiv:1409.8005 [hep-ph]].

[26] G. C. Branco, I. de Medeiros Varzielas and S. F. King, Phys. Rev. D 92 (2015) 036007 [arXiv:1502.03105 [hep-ph]]; G. C. Branco, I. de Medeiros Varzielas and S. F. King, Nucl. Phys. B 899 (2015) 14 [arXiv:1505.06165 [hep-ph]].

[27] Y. Shimizu, M. Tanimoto and A. Watanabe, Prog. Theor. Phys. 126 (2011) 81 [arXiv:1105.2929].

[28] S. F. King and C. Luhn, JHEP 1109 (2011) 042 [arXiv:1107.5332].

[29] D. Hernandez and A. Y. Smirnov, Phys. Rev. D 86 (2012) 053014 [arXiv:1204.0445]; D. Hernandez and A. Y. Smirnov, Phys. Rev. D 87 (2013) 5, 053005 [arXiv:1212.2149].

[30] S. F. King, Phys. Lett. B 659 (2008) 244 [arXiv:0710.0530 [hep-ph]].

[31] S. Pakvasa, W. Rodejohann and T. J. Weiler, Phys. Rev. Lett. 100 (2008) 111801 [arXiv:0711.0052 [hep-ph]].

[32] P. Ballett, S. F. King, C. Luhn, S. Pascoli and M. A. Schmidt, Phys. Rev. D 89 (2014) 016016 [arXiv: 1308.4314].

[33] S. F. King, JHEP 0508 (2005) 105 [hep-ph/0506297].

[34] I. Masina, Phys. Lett. B 633 (2006) 134 [hep-ph/0508031].

[35] S. Antusch and S. F. King, Phys. Lett. B 631 (2005) 42 [hep-ph/0508044]; S. Antusch, P. Huber, S. F. King and T. Schwetz, JHEP 0704 (2007) 060 [hep-ph/0702286].

[36] P. Ballett, S. F. King, C. Luhn, S. Pascoli and M. A. Schmidt, arXiv:1410.7573 [hep-ph]. 
[37] D. Marzocca, S. T. Petcov, A. Romanino and M. C. Sevilla, JHEP 1305 (2013) 073 [arXiv:1302.0423]; S. T. Petcov, arXiv:1405.6006.

[38] I. Girardi, S. T. Petcov and A. V. Titov, arXiv:1410.8056 [hep-ph].

[39] S. Antusch, S. F. King, C. Luhn and M. Spinrath, Nucl. Phys. B 856 (2012) 328 [arXiv:1108.4278].

[40] S. F. King, JHEP 1307 (2013) 137 [arXiv:1304.6264]; S. F. King, Phys. Lett. B 724 (2013) 92 [arXiv:1305.4846].

[41] S. F. King, JHEP 1401 (2014) 119 [arXiv:1311.3295 [hep-ph]];

[42] F. Bjorkeroth and S. F. King, arXiv:1412.6996 [hep-ph].

[43] S. F. King, JHEP 1408 (2014) 130 [arXiv:1406.7005 [hep-ph]]; S. F. King, JHEP 1401 (2014) 119 [arXiv:1311.3295 [hep-ph]].

[44] C. Beasley, J. J. Heckman and C. Vafa, "GUTs and Exceptional Branes in F-theory - II: Experimental Predictions," JHEP 0901 (2009) 059 [arXiv:0806.0102 [hep-th]].

[45] G. K. Leontaris, PoS CORFU 2011 (2011) 095 [arXiv:1203.6277 [hep-th]].

[46] I. Antoniadis and G. K. Leontaris, Eur. Phys. J. C 73 (2013) 2670 [arXiv:1308.1581 [hep-th]].

[47] A. Karozas, S. F. King, G. K. Leontaris and A. Meadowcroft, JHEP 1409 (2014) 107 [arXiv:1406.6290 [hep-ph]]; A. Karozas, S. F. King, G. K. Leontaris and A. K. Meadowcroft, arXiv:1505.00937 [hep-ph]. 